\title{
HALL CURRENT EFFECT ON VISCOELASTIC (WALTER'S LIQUID MODEL-B) MHD OSCILLATORY CONVECTIVE CHANNEL FLOW THROUGH A POROUS MEDIUM WITH HEAT RADIATION
}

\author{
Balvinder Pal Garg ${ }^{1}$, Krishan Dev Singh ${ }^{2}$ and A. K. Bansal ${ }^{3}$ \\ ${ }^{1}$ Devraj Group’s Technical Campus, Ferozpur, Punjab, India \\ ${ }^{2}$ Wexlow Building, Lower Kaithu, Shimla 171003, India \\ E-mail: kdsinghshimla@gmail.com \\ ${ }^{3}$ Research Scholar, Punjab Technical University, Jalandhar, Punjab, India
}

(Received June 23, 2013)

\begin{abstract}
An oscillatory MHD convective flow of an incompressible, viscoelastic (Walter's liquid model-B) and electrically conducting fluid through porous medium filled in a vertical porous channel is analyzed. The two porous plates of the channel are subjected to a constant injection and suction velocity as shown in Fig.1. A magnetic field of uniform strength is applied perpendicular to the plates of the channel. The temperature of one of the plates varies periodically and the temperature difference between the two plates is high enough to induce the heat due to radiation. A closed form solution of the purely oscillatory flow is obtained. The velocity, temperature and the skin-friction in terms of its amplitude and phase angle have been shown graphically to observe the effects of viscoelasticity $\gamma$, injection/suction parameter $\lambda$, Grashof number Gr, Hartmann number M, Hall parameter $\mathrm{H}$, the pressure A, Prandtl number Pr, Radiation parameter N and the frequency of oscillation $\omega$.
\end{abstract}

Key words: Injection/suction, viscoelastic (Walter's liquid model-B), convection, magnetohydromagnetic (MHD), oscillatory, radiation.

\section{INTRODUCTION}

The study of the flows of visco-elastic fluids is important in the fields of petroleum technology and in the purification of crude oils. In recent years, flows of visco-elastic fluids attracted the attention of several scholars in view of their practical and fundamental importance associated with many industrial applications. Literature is replete with the various flow problems considering variety of geometries such as RAJGOPAL [23], RAJGOPAL and GUPTA [24] ARIEL [3], POP and GORLA [22]. HAYAT et al. [15] discussed periodic unsteady flows of a non-Newtonian fluid. CHOUDHURY and DAS [10] studied the oscillatory viscoelastic flow in a channel filled with porous medium in the presence of radiative heat transfer.

The flow problems of electrically conducting fluids are currently receiving considerable attention. The magnetohydrodynamic (MHD) flows has many practical applications such as electromagnetic flow meters, electromagnetic pumps and hydromagnetic generators etc. The interest in MHD convective flows with heat transfer is renewed due to its importance in the design of MHD generators and accelerators in geophysics, in underground water and energy 
storage systems. Several scholars have shown their interest in studying MHD and heat transfer flows in porous and non-porous media. The effect of transversely applied magnetic field on convection flows of an electrically conducting fluid has been discussed by several authors notably Nigam and Singh [21], SoundalgeKar and Bhat [33], VajRaVelu [35], AtTia and Котв [8] etc.

When the strength of the magnetic field is strong enough then one cannot neglect the effects of Hall currents. Even though it is of considerable importance to study how the results of the hydrodynamical problems get modified by the effects of Hall currents. A comprehensive discussion of Hall currents is given by COWLING [13]. SOUNDALGEKAR [32] studied the Hall and Ion-slip effects in MHD Couette flow with heat transfer. SoUNDALGEKAR and UPLEKAR [34] also analyzed Hall effects in MHD Couette flow with heat transfer. HOSSAIN and RASHID [16] investigate Hall effect on hydromagnetic free convection flow along a porous flat plate with mass transfer. ATTIA [5] studied Hall current effects on the velocity and temperature fields on an unsteady Hartmann flow. Effects of Hall currents on free convective flow past an accelerated vertical porous plate in a rotating system with heat source /sink is analyzed by SINGH and GARG [30].

The fluid flow through porous medium is another important aspect which has attracted the attention of scientists and engineers because of its usefulness in the fields of agricultural engineering to study the underground water resources, seepage of water in river beds, in chemical engineering for filtration and purification processes. RAPTIS et al. [27] studied hydromagnetic free convection flow through porous medium between two parallel plates. RAPTIS and PERDIKIS [26] analyzed oscillatory flow through porous medium by the presence of free convection flow. HOSSANIEN and MANSOUR [14] investigated unsteady magnetic flow through a porous medium between two infinite parallel plates. AlAGOA et al. [1] studied the radiative and free convective effects of a MHD flow through a porous medium between infinite parallel plates with time-dependent suction. Taking into account the heat radiation and the Hall currents SINGH et al. [31] studied heat and mass transfer in an unsteady MHD free convective flow through a porous medium bounded by vertical porous channel.

The flows of viscoelastic fluids through porous medium are very important particularly in the fields of petroleum technology for the flow of oil through porous rocks, in chemical engineering and in the cases like drug permeation through human skin. ALDOSS et al. [2] studied MHD mixed convection flow from a vertical plate embedded in porous medium. RAJGOPAL et al. [25] analyzed oscillatory flow of an electrically conducting viscoelastic fluid over a stretching sheet in a saturate porous medium. ATTIA and EwIS [4] investigated an unsteady MHD Couette flow with heat transfer of a viscoelastic fluid under exponential decaying pressure gradient. SINGH [29] analyzed an oscillatory mixed convection flow of a viscoelastic electrically conducting fluid in an infinite vertical channel filled with porous medium. Considering the Hall effects ATTIA [6] discussed unsteady Hartmann flow of a viscoelastic fluid. CHOUDHARY and JHA [7] analyzed heat and mass transfer in elastic-viscous fluid past an impulsively started infinite vertical plate with Hall current. Very recently, SINGH [17] investigated MHD mixed convection visco-elastc slip-flow through a porous medium in a vertical porous channel with thermal radiation.

The object of the present paper is to study Hall current effect on the oscillatory MHD convective flow of a viscoelastic (Walter's liquid-B) fluid through a porous medium filled in a vertical channel. Constant injection and suction is applied at the left and the right infinite porous plates respectively. A uniform magnetic field is applied along the axis perpendicular to the planes of the plates. This applied transverse magnetic field is strong enough so that the Hall currents are induced. The temperature difference between the plates of the channel is sufficiently high to induce heat radiation. A general exact solution of the partial differential equations governing the flow problem is obtained and the effects of various flow parameters on the 
velocity field and the skin friction are discussed in the last section of the paper with the help of figures.

\section{BASIC EQUATIONS}

In order to derive the basic equations for the problem under consideration following assumptions are made:

(i) The two infinite vertical parallel plates of the channel are permeable and electrically non-conducting.

(ii) The vertical channel is filled with a porous medium.

(iii) The flow considered is fully developed, laminar and oscillatory.

(iv) The fluid is viscoelastic (Walter's liquid model-B), incompressible and finitely conducting.

(v) All fluid properties are assumed to be constant except that of the influence of density variation with temperature is considered only in the body force term.

(vi) The pressure gradient in the channel oscillates periodically with time.

(vii) A magnetic field of uniform strength $B_{0}$ is applied perpendicular to the plates of the channel.

(viii) The temperature of a plate is non-uniform and oscillates periodically with time.

(ix) The temperature difference of the two plates is also assumed to be high enough to induce heat transfer due to radiation.

(x) The fluid is assumed to be optically thin with relatively low density.

Under these assumptions we write the equations governing the flow as: Equation of Continuity:

$\nabla \cdot V=0$.

Momentum Equation:

$\frac{\partial \boldsymbol{V}}{\partial t}+(\boldsymbol{V} \cdot \nabla \boldsymbol{V})=-\frac{1}{\rho} \nabla p^{*}+\vartheta_{1} \nabla^{2} \boldsymbol{V}+\frac{\boldsymbol{\vartheta}_{1}}{\boldsymbol{K}^{*}} \boldsymbol{V}+\nabla \cdot \exists+\frac{1}{\rho}(\boldsymbol{J} \times \boldsymbol{B})+\boldsymbol{F}$.

Energy Equation:

$\rho c_{p}\left[\frac{\partial T}{\partial t}+(\boldsymbol{V} . \nabla) T\right]=k \nabla^{2} T-\nabla q$.

Kirchhoff's First Law:

$$
\operatorname{div} \boldsymbol{J}=0 \text {. }
$$

General Ohm's Law:

$$
\boldsymbol{J}+\frac{\omega_{e} \tau_{e}}{B_{0}}(\boldsymbol{J} \times \boldsymbol{B})=\sigma\left[\boldsymbol{E}+\boldsymbol{V} \times \boldsymbol{B}+\frac{1}{e \eta_{e}} \nabla p_{e}\right] .
$$

Gauss's Law of Magnetism:

$$
\operatorname{div} \boldsymbol{B}=0 \text {. }
$$

where $\boldsymbol{V}$ is the velocity vector, p is the pressure, $\rho$ is the density, $\boldsymbol{B}$ is the magnetic induction vector, $\boldsymbol{J}$ is the current density, $\mu$ is the coefficient of viscosity, $\vartheta_{1}$ is the kinematic viscosity, $\mathrm{t}^{*}$ is the time, $\mathrm{g}$ is the acceleration due to gravity, $\beta$ is the coefficient of volume expansion, $\mathrm{K}^{*}$ is the permeability of the porous medium, $\mathrm{C}_{\mathrm{p}}$ is the specific heat at constant pressure, $\mathrm{T}^{*}$ is the temperature, $\mathrm{k}$ is the thermal conductivity, $\mathrm{q}$ is the radiative heat, $\sigma$ is the electrical conductivity, $\mathrm{e}$ is the electron charge, $\omega_{\mathrm{e}}$ is the electron frequency, $\tau_{\mathrm{e}}$ is the electron collision time, $\mathrm{p}_{\mathrm{e}}$ is the electron pressure, $\boldsymbol{E}$ is the electric field and $\eta_{\mathrm{e}}$ is the number density of electron, $\exists$ is the Cauchy stress tensor and the constitutive equation derived by COLEMAN and NOLL [12] for an incompressible homogeneous Walter' liquid-B is 


$$
\exists=-p_{1} I+\mu_{1} A_{1}+\mu_{2} A_{2}+\mu_{3} A_{1}^{2} .
$$

Here $-p_{1} I$ is the interdeterminate part of the stress due to constraint of incompressibility, $\mu_{1}, \mu_{2}$ and $\mu_{3}$ are the material constants describing viscosity, elasticity and cross-viscosity respectively. The kinematic $A_{1}$ and $A_{2}$ are the Rivelen Ericson constants defined as

$$
A_{1}=(\nabla \bar{V})+(\nabla \bar{V})^{T}, A_{2}=\frac{d A_{1}}{d t}+(\nabla \bar{V})^{T} A_{1}+A_{1}(\nabla \bar{V})
$$

where $\nabla$ denotes the gradient operator and $\mathrm{d} / \mathrm{dt}$ the material time derivative. According to MARKOVITZ and COLEMAN [19] the material constants $\mu_{1}, \mu_{3}$ are taken as positive and $\mu_{2}$ as negative.

\section{FORMULATION OF THE PROBLEM}

In the present analysis we consider an unsteady flow of a viscoelastic (Walter' liquid model- B), incompressible and electrically conducting fluid bounded by two infinite vertical porous plates distance ' $d$ ' apart. A coordinate system is chosen such that the $X^{*}$-axis is oriented upward along the centerline of the channel and $\mathrm{Z}^{*}$-axis taken perpendicular to the planes of the plates lying in $z^{*}= \pm \frac{d}{2}$ planes. The fluid is injected through the porous plate at $z^{*}=-\frac{d}{2}$ with constant velocity $\mathrm{w}_{0}$ and simultaneous sucked through the other porous plate at $z^{*}=+\frac{d}{2}$ with the same velocity $\mathrm{w}_{0}$. The non-uniform temperature of the plate at $z^{*}=+\frac{d}{2}$ is assumed to be varying periodically with time. The temperature difference between the plates is high enough to induce the heat due to radiation. A transverse magnetic field of uniform strength $\mathbf{B}\left(0,0, \mathbf{B}_{0}\right)$ is also applied with $\mathrm{B}_{0}$ component along the $\mathrm{Z}^{*}$ - axis which is considered perpendicular to the planes of the plates. All physical quantities depend on $\mathrm{z}^{*}$ and $\mathrm{t}^{*}$ only for this problem of fully developed laminar flow. A schematic diagram of the flow problem is shown in figure 1. Since the porous plates are subjected to constant injection/suction velocity $w_{0}$, thus, equation of continuity $\nabla . \boldsymbol{V}=0$ (1) gives on integration $w^{*}=w_{0}$. Then the velocity may reasonably be assumed with its components along $\mathrm{x}^{*}, \mathrm{y}^{*}, \mathrm{z}^{*}$ directions as $\mathbf{V}\left(\mathrm{u}^{*}, \mathrm{v}^{*}, \mathrm{w}_{0}\right)$. Also the equation (6) for the magnetic field $\vec{B}=\left(B_{x}^{*}, B_{y}^{*}, B_{z}^{*}\right)$ gives $B_{z}^{*}=B_{0}$ (constant).

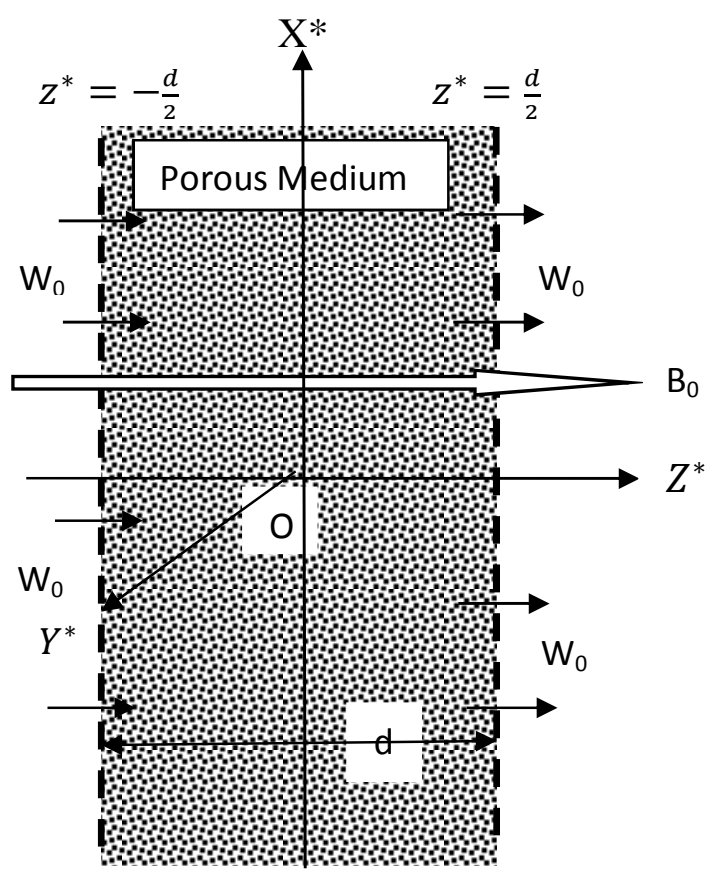

Fig. 1. Physical configuration of the flow. 
$\left(j_{x}^{*}, j_{y}^{*}, j_{z}^{*}\right)$ are the components of electric current density $\boldsymbol{J}$. The equation of conservation of electric charge (4) gives $j_{z}^{*}=$ constant.

For non-conducting plates $j_{z}^{*}=0$

at the plates and hence zero everywhere in the fluid. Under the usual assumptions that the electron pressure (for a weakly ionized gas), the thermoelectric pressure, ion slip and the external electric field arising due to polarization of charges is negligible. It is assumed that no applied and polarization voltage exists. This corresponds to the case where no energy is being added or extracted from the fluid by electrical means (MEYER [20]) i.e. electrical field $\boldsymbol{E}=0$. Therefore, equation (5) takes the form:

$$
\vec{\jmath}+\frac{\omega_{e} \tau_{e}}{B_{0}}(\vec{\jmath} \times \vec{B})=\sigma(\vec{V} \times \vec{B})
$$

After using equation (8), equation (9) in component form becomes

$$
\begin{aligned}
& j_{x}^{*}+\omega_{e} \tau_{e} j_{y}^{*}=\sigma B_{0} v^{*} \\
& j_{y}^{*}-\omega_{e} \tau_{e} j_{x}^{*}=-\sigma B_{0} u^{*}
\end{aligned}
$$

Solving (10) and (11) for $j_{x}^{*}$ and $j_{y}^{*}$, we get

$$
j_{x}^{*}=\frac{\sigma B_{0}}{\left(1+H^{2}\right)}\left(H u^{*}+v^{*}\right) \quad \text { and } \quad j_{y}^{*}=\frac{\sigma B_{0}}{\left(1+H^{2}\right)}\left(H v^{*}-u^{*}\right)
$$

where $H=\omega_{e} \tau_{e}$ is the Hall parameter.

Following AtTia and Ewis [4], AtTia [7], Kumar and KHEM [18], and under the usual Boussinesq approximation the momentum equation (2) reduces to

$$
\begin{gathered}
\frac{\partial u^{*}}{\partial t^{*}}+w_{0} \frac{\partial u^{*}}{\partial z^{*}}=-\frac{1}{\rho} \frac{\partial p^{*}}{\partial x^{*}}+\vartheta_{1} \frac{\partial^{2} u^{*}}{\partial z^{* 2}}+\vartheta_{2} \frac{\partial^{3} u^{*}}{\partial z^{* 2} \partial t^{*}}+\frac{\sigma B_{0}^{2}\left(H v^{*}-u^{*}\right)}{\rho\left(1+H^{2}\right)}-\frac{v u^{*}}{K^{*}}+g \beta\left(T^{*}-T_{1}\right) \\
\frac{\partial v^{*}}{\partial t^{*}}+w_{0} \frac{\partial v^{*}}{\partial z^{*}}=-\frac{1}{\rho} \frac{\partial p^{*}}{\partial y^{*}}+\vartheta_{1} \frac{\partial^{2} v^{*}}{\partial z^{* 2}}+\vartheta_{2} \frac{\partial^{3} v^{*}}{\partial z^{* 2} \partial t^{*}}-\frac{\sigma B_{0}^{2}\left(H u^{*}+v^{*}\right)}{\rho\left(1+H^{2}\right)}-\frac{v u^{*}}{K^{*}} \\
0=-\frac{1}{\rho} \frac{\partial p^{*}}{\partial z^{*}}, \\
\text { (14) } \quad \frac{\partial T^{*}}{\partial t^{*}}+w_{0} \frac{\partial T^{*}}{\partial z^{*}}=\frac{k}{\rho c_{p}} \frac{\partial^{2} T^{*}}{\partial z^{* 2}}-\frac{1}{\rho c_{p}} \frac{\partial q}{\partial z^{*}}
\end{gathered}
$$

where $\vartheta_{2}$ is viscoelasticity and the last term in equation (15) is the radiative heat flux.

Following COGLEY et al [11] it is assumed that the fluid is optically thin with a relatively low density and the heat flux due to radiation in equation (15) is given by

$$
\frac{\partial q}{\partial z^{*}}=4 \alpha^{2}\left(T^{*}-T_{1}\right)
$$

where $\alpha$ is the mean radiation absorption coefficient. After the substitution of equation (16), equation (15) becomes

$$
\frac{\partial T^{*}}{\partial t^{*}}+w_{0} \frac{\partial T^{*}}{\partial z^{*}}=\frac{k}{\rho c_{p}} \frac{\partial^{2} T^{*}}{\partial z^{* 2}}-\frac{4 \alpha^{2}}{\rho c_{p}}\left(T^{*}-T_{1}\right) .
$$

Equation (14) shows the constancy of the hydrodynamic pressure along the axis of rotation. We shall assume now that the fluid flows under the influence of pressure gradient varying periodically with time in the $\mathrm{X}^{*}$-axis is of the form

$$
-\frac{1}{\rho} \frac{\partial p^{*}}{\partial x^{*}}=A \cos \omega^{*} t^{*} \text { and }-\frac{1}{\rho} \frac{\partial p^{*}}{\partial y^{*}}=0, \text { where } \mathrm{A} \text { is a constant. }
$$

The boundary conditions for the problem are

$$
\begin{gathered}
z^{*}=\frac{d}{2}: u^{*}=v^{*}=0, T^{*}=T_{1}+\left(T_{2}-T_{1}\right) \cos \omega^{*} t^{*}, \\
z^{*}=-\frac{d}{2}: u^{*}=v^{*}=0, T^{*}=T_{1},
\end{gathered}
$$

where $\omega^{*}$ is the frequency of oscillations.

Introducing the following non-dimensional quantities: 
$\eta=\frac{z^{*}}{d}, x=\frac{x^{*}}{d}, y=\frac{y^{*}}{d}, u=\frac{u^{*}}{w_{0}}, v=\frac{v^{*}}{w_{0}}, \quad T=\frac{T^{*}-T_{1}}{T_{2}-T_{1}}, t=\frac{t^{*} w_{0}}{d}, \omega=\frac{\omega^{*} d}{w_{0}} \quad p=\frac{p^{*}}{\rho w_{0}^{2}}$,

into equations (5), (6) and (10), we get

$$
\begin{aligned}
& \lambda\left(\frac{\partial u}{\partial t}+\frac{\partial u}{\partial \eta}\right)=-\lambda \frac{\partial p}{\partial x}+\frac{\partial^{2} u}{\partial \eta^{2}}+\gamma \frac{\partial^{3} u}{\partial \eta^{2} \partial t}+\frac{M^{2}(H v-u)}{\left(1+H^{2}\right)}-K^{-1} u+G r T, \\
& \lambda\left(\frac{\partial v}{\partial t}+\frac{\partial v}{\partial \eta}\right)=-\lambda \frac{\partial p}{\partial y}+\frac{\partial^{2} v}{\partial \eta^{2}}+\gamma \frac{\partial^{3} v}{\partial \eta^{2} \partial t}-\frac{M^{2}(H u+v)}{\left(1+H^{2}\right)}-K^{-1} v, \\
& \lambda P r\left(\frac{\partial T}{\partial t}+\frac{\partial T}{\partial \eta}\right)=\frac{\partial^{2} T}{\partial \eta^{2}}-N^{2} T,
\end{aligned}
$$

where ' $*$ ' represents the dimensional physical quantities,

$\lambda=\frac{w_{0} d}{\vartheta_{1}}$ is the injection/suction parameter,

$\gamma=\frac{v_{2} \lambda}{d^{2}}$ is the visco-elastic parameter,

$M=B_{0} d \sqrt{\frac{\sigma}{\rho \vartheta_{1}}}$ is the Hartmann number,

$H=\omega_{e} \tau_{e}$ is the Hall parameter,

$K=\frac{K^{*}}{d^{2}}$ is the permeability of the porous medium,

$G r=\frac{g \beta d^{2}\left(T_{2}-T_{1}\right)}{\vartheta_{1} w_{0}}$ is the Grashof number,

$\operatorname{Pr}=\frac{\rho \vartheta_{1} c_{p}}{k}$ is the Prandtl number,

$N=\frac{2 \alpha d}{\sqrt{k}}$ is the radiation parameter,

$\omega=\frac{\omega^{*} d}{w_{0}}$ is the frequency of oscillations.

The boundary conditions in the dimensionless form become

$$
\begin{aligned}
& \eta=\frac{1}{2}: \quad u=v=0, \quad T=\cos \omega t \\
& \eta=-\frac{1}{2}: \quad u=v=0, \quad T=0 .
\end{aligned}
$$

For the oscillatory internal flow we shall assume that the fluid flows under the influence of a non-dimension pressure gradient varying periodically with time in the direction of X-axis only which implies that

$$
-\frac{\partial p}{\partial x}=A \cos \omega t, \text { and }-\frac{\partial p}{\partial y}=0
$$

\section{SOLUTION OF THE PROBLEM}

Now combining equations (22) and (23) into single equation by introducing a complex function of the form $\mathrm{F}=\mathrm{u}+\mathrm{iv}$ and with the help of equation (27), we get

$$
\lambda\left(\frac{\partial F}{\partial t}+\frac{\partial F}{\partial \eta}\right)=-\lambda \frac{\partial p}{\partial x}+\frac{\partial^{2} F}{\partial \eta^{2}}+\gamma \frac{\partial^{3} F}{\partial \eta^{2} \partial t}-\left(\frac{M^{2}(1+i H)}{\left(1+H^{2}\right)}+K^{-1}\right) F+G r T
$$

with corresponding boundary conditions as

$$
\begin{aligned}
& \eta=\frac{1}{2}: \quad F=0, \quad T=\cos \omega t, \\
& \eta=-\frac{1}{2}: \quad F=0, \quad T=0 .
\end{aligned}
$$

In order to solve equation (28) and (24) under boundary conditions (29) and (30) it is convenient to adopt complex notations for the velocity, temperature and the pressure as under:

$$
F(\eta, t)=F_{0}(\eta) e^{i \omega t}, \quad T=\theta_{0}(\eta) e^{i \omega t},-\frac{\partial y}{\partial x}=A e^{i \omega t} .
$$

The solutions will be obtained in terms of complex notations, the real part of which will have physical significance. 
The boundary conditions (29) and (30) in complex notations can also be written as

$$
\begin{aligned}
& \eta=\frac{1}{2}: \quad F=0, \quad T=e^{i \omega t}, \\
& \eta=-\frac{1}{2}: \quad F=0, \quad T=0 .
\end{aligned}
$$

Substituting expressions (31) in equations (28) and (24), we get

$$
\begin{aligned}
& (1+i \omega \gamma) \frac{d^{2} F_{0}}{d \eta^{2}}-\lambda \frac{d F_{0}}{d \eta}-\left\{\frac{M^{2}(1+i H)}{\left(1+H^{2}\right)}+K^{-1}+i \omega \lambda\right\} F_{0}=-\lambda A-G r \theta_{0}, \\
& \frac{d^{2} \theta_{0}}{d \eta^{2}}-\lambda \operatorname{Pr} \frac{d \theta_{0}}{d \eta}-\left(N^{2}+i \omega \lambda P r\right) \theta_{0}=0 .
\end{aligned}
$$

The transformed boundary conditions reduce to

$$
\begin{aligned}
\eta=\frac{1}{2}: & F_{0}=0, \quad \theta_{0}=1, \\
\eta=-\frac{1}{2}: & F_{0}=0, \quad \theta_{0}=0 .
\end{aligned}
$$

The solution of the ordinary differential equation (34) under the boundary conditions (36) and (37) gives the velocity field as

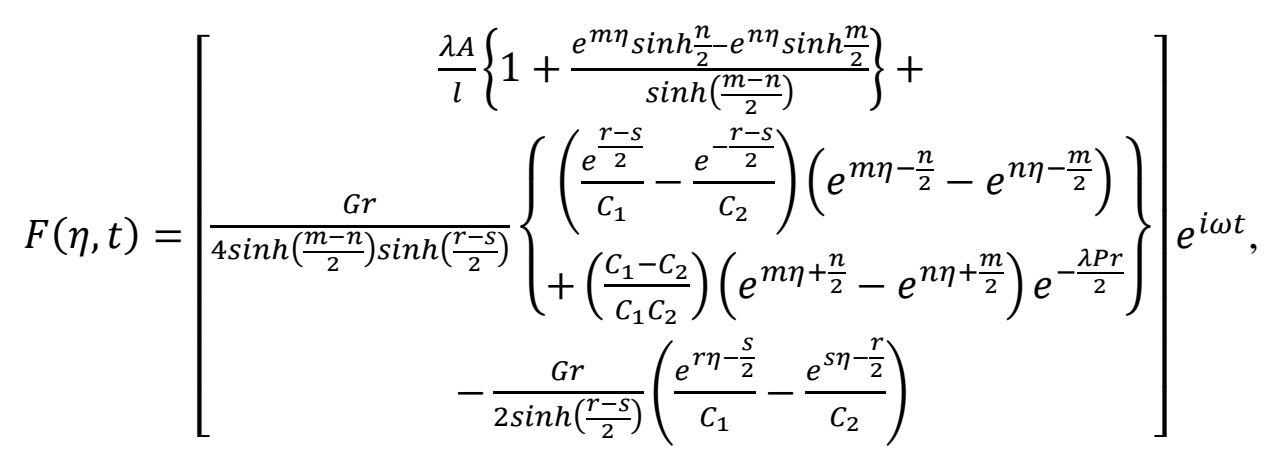

where $l=\left\{\frac{M^{2}(1+i H)}{\left(1+H^{2}\right)}+K^{-1}+i \omega \lambda\right\}, C_{1}=(1+i \omega \gamma) r^{2}-\lambda r-l, C_{2}=(1+i \omega \gamma) s^{2}-\lambda s-l$

$$
\begin{array}{ll}
m=\frac{\lambda+\sqrt{\lambda^{2}+4 l(1+i \omega \gamma)}}{2(1+i \omega \gamma)}, & n=\frac{\lambda-\sqrt{\lambda^{2}+4 l(1+i \omega \gamma)}}{2(1+i \omega \gamma)}, \\
r=\frac{\lambda P r+\sqrt{\lambda^{2} P r^{2}+4\left(N^{2}+i \omega \lambda P r\right)}}{2}, & s=\frac{\lambda P r-\sqrt{\lambda^{2} P r^{2}+4\left(N^{2}+i \omega \lambda P r\right)}}{2} .
\end{array}
$$

Similarly, the solution of equation (35) for the temperature field can be obtained under the boundary conditions (36) and (37) as

$$
T(\eta, t)=\left(\frac{e^{r \eta-\frac{s}{2}}-e^{s \eta-\frac{r}{2}}}{2 \sinh \left(\frac{r-s}{2}\right)}\right) e^{i \omega t} .
$$

From the velocity field obtained in equation (38) we can get the skin-friction $\tau_{L}$ at the left plate $(\eta=-0.5)$ in terms of its amplitude $|F|$ and phase angle $\varphi$ as

$$
\tau=|\mathrm{F}| \cos (\omega \mathrm{t}+\varphi), \text { with }
$$




$$
F=F_{r}+i F_{i}=\left[\begin{array}{c}
\frac{\lambda A}{l}\left(\frac{m e^{-\frac{m}{2} \sinh \frac{n}{2}-n e^{-\frac{n}{2}} \sinh \frac{m}{2}}}{\sinh \left(\frac{m-n}{2}\right)}\right)+ \\
\frac{G r}{4 \sinh \left(\frac{m-n}{2}\right) \sinh \left(\frac{r-s}{2}\right)}\left\{\begin{array}{c}
\left(\frac{e^{\frac{r-s}{2}}}{C_{1}}-\frac{e^{-\frac{r-s}{2}}}{C_{2}}\right)(m-n) e^{-\frac{\lambda}{2(1+i \omega \gamma)}}+ \\
\left(\frac{C_{1}-C_{2}}{C_{1} C_{2}}\right)\left(m e^{-\frac{m-n}{2}}-n e^{\frac{m-n}{2}}\right) e^{-\frac{\lambda P r}{2}}
\end{array}\right\} \\
-\frac{G r}{2 \sinh \left(\frac{r-s}{2}\right)}\left(\frac{r}{C_{1}}-\frac{s}{C_{2}}\right) e^{-\frac{\lambda P r}{2}}
\end{array}\right] .
$$

The amplitude is $|\mathrm{F}|=\sqrt{\mathrm{F}_{\mathrm{r}}^{2}+\mathrm{F}_{\mathrm{i}}^{2}}$ and the phase angle is $\varphi=\tan ^{-1} \frac{\mathrm{F}_{\mathrm{i}}}{\mathrm{Fr}_{\mathrm{r}}}$.

Similarly the Nusselt number $\mathrm{Nu}$ in terms of its amplitude $|H|$ and the phase angle $\psi$ can be obtained from the solution (39) for the temperature field as

$q=|H| \cos (\omega t+\psi)$,
with $=H r+i H i=\frac{(r-s) e^{-\frac{\lambda P r}{2}}}{2 \sinh \left(\frac{r-s}{2}\right)}$,

where the amplitude $|H|$ and the phase angle $\psi$ of the rate of heat transfer are given as

$$
|H|=\sqrt{H r^{2}+H i^{2}}, \psi=\tan ^{-1} \frac{H i}{H r} .
$$

The temperature field, amplitude and phase of the Nusselt number need no further discussion because these have already been discussed in detail by SINGH [28].

\section{DISCUSSION}

An analytical solution of the problem of MHD convection flow of a viscoelastic fluid through a porous medium bounded by two infinite vertical porous plates when the pressure gradient varies periodically with time is obtained. The two porous plates are subjected to constant injection and suction velocity. Numerical evaluations of the analytical results obtained in the previous section are then illustrated through figures. The influence of each of the parameters on the velocity profiles, the amplitude and the phase of the skin-friction are depicted through these figures.

Figure 2 shows the variations of the velocity over the width of the channel. This figure clearly shows that the velocity is maximal in the middle of the channel which leads to parabolic velocity profiles in the channel as expected. The effects of viscoelastic parameter $\gamma$ injection/suction parameter $\lambda$, Grashof number Gr, Hartmann number $M$, Hall parameter $H$, permeability of the porous medium K, Prandtl number Pr, radiation parameter N, favorable pressure gradient $\mathrm{A}$ and the frequency of oscillations $\omega$ on the velocity are presented in Fig.2. Each curve is compared with the dotted curve I (---). It is observed that the velocity goes on decreasing (curves I \& II) with the increase of the viscoelstic parameter $\gamma$. i.e. the flow retards with the increasing viscoelastic parameter. Curves I \& III in this figure clearly show that there is a sharp rise in the velocity with the increase of the injection/suction parameter $\lambda$. From curves I \& IV it is inferred that the velocity also increases as the Grashof number Gr increases from 1 to 5. Physically it means that the enhancement of the buoyancy force leads to increase the velocity. A decrease in the velocity is also observed with the increase of Hartmann number (curves I \& V). This means that the increasing transverse magnetic field strength induce a drag force which tends to resist the fluid flow. The velocity increases with the increase of Hall current parameter $\mathrm{H}$ (curves I \& VI). Comparison of curves I and VII reveal that the velocity decreases slightly with the increasing permeability of the porous medium for the viscoelastic fluid flow. Since the 
Prandtl number gives the relative importance of viscous dissipation to the thermal dissipation so for larger Prandtl number viscous dissipation is predominant and due to this velocity decreases (curves I \& VIII). Thus, the velocity in the case of water $(\mathrm{Pr}=7)$ as the fluid is less than that in the case of air $(\operatorname{Pr}=0.7)$. Retaining the values of all of the parameters fixed and increasing only the value of the radiation parameter from 1 to 5 indicates that the velocity decreases (curves I \& IX). This figure also reveals (curves I \& X) that as the favorable pressure gradient in the channel is increased the velocity increases rapidly. As expected it is due to the fact that the flow for larger pressure gradient in the channel is faster. The velocity decreases significantly (curves I \& $\mathrm{XI}$ ) when the frequency of oscillations $\omega$ is increased keeping rest of the parameters fixed.

The variations of the amplitude $|\mathrm{F}|$ and the phase $\varphi$ of the skin-friction with the increase of different parameters are presented in Fig. 3a and $3 \mathrm{~b}$ respectively. It is obvious from Fig. 3a that for any set of parameters the amplitude goes on decreasing with the increasing frequency of oscillations $\omega$. The increase in the skin-friction amplitude is noticed with the increase of injection/suction parameter $\lambda$, Grashof number $\mathrm{Gr}$, the Hall parameter $\mathrm{H}$, the permeability of the porous medium and the pressure gradient A. It is true physically also because the increase in these parameters results into velocity increase which consequently leads to the enhancement of frictional force. However, the increase in viscoelastic parameter $\gamma$, Hartmann number M, Prandtl number and the radiation parameter attribute towards the decrease in the amplitude of the skinfriction.

The behavior of the phase angle $\varphi$ of the skin-friction $\tau$ is shown in Fig. $3 \mathrm{~b}$ for different values of various flow parameters. From this figure it is evident that there is always a phase lag because the values of $\varphi$ computed numerically remain negative throughout for any set of values of the flow parameters. We notice from Fig. $3 \mathrm{~b}$ that the phase lag increases with the increase of viscoelastic parameter, Hartmann number and the permeability of the porous medium. However, the phase lag decreases with the increase of Prandtl number, the radiation parameter and the pressure gradient. This variation with the Prandtl number indicates that the phase lag is less in water $(\operatorname{Pr}=7.0)$ than in air $(\operatorname{Pr}=0.7)$. The opposing effects of the Grashof number and the Hall parameter are observed as the frequency increase.

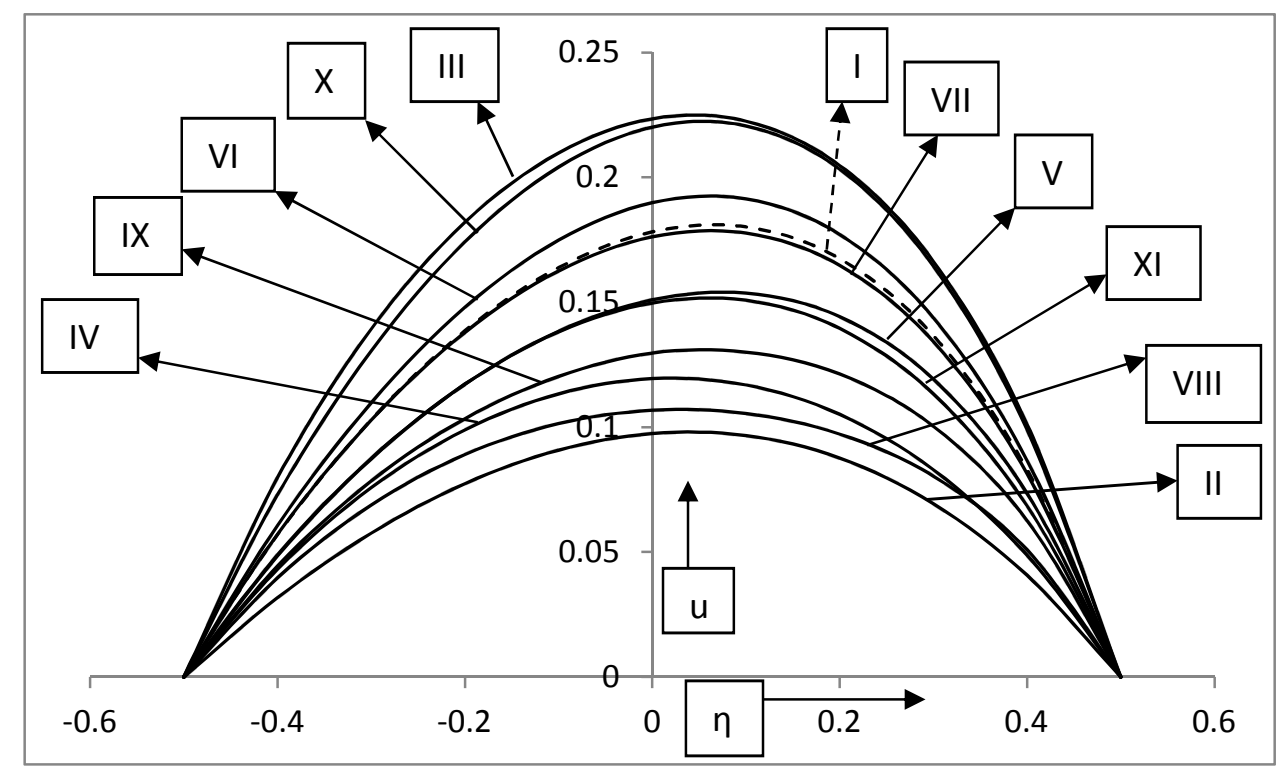

Fig. 2. Velocity Profiles. 
Table 1. Values of parameters plotted in Fig. 2.

$\gamma \lambda$ Gr MH K $\operatorname{Pr} \quad \mathrm{N}$ A $\omega$

$\begin{array}{lllllllllll}0.2 & 0.5 & 5 & 2 & 1 & 0.2 & 0.7 & 1 & 5 & 5 & \mathrm{I}(---)\end{array}$

$\begin{array}{lllllllllll}0.4 & 0.5 & 5 & 2 & 1 & 0.2 & 0.7 & 1 & 5 & 5 & \text { II }\end{array}$

$\begin{array}{llllllllllll}0.2 & 1.0 & 5 & 2 & 1 & 0.2 & 0.7 & 1 & 5 & 5 & \text { III }\end{array}$

$\begin{array}{lllllllllll}0.2 & 0.5 & 1 & 2 & 1 & 0.2 & 0.7 & 1 & 5 & 5 & \text { IV }\end{array}$

$\begin{array}{lllllllllll}0.2 & 0.5 & 5 & 3 & 1 & 0.2 & 0.7 & 1 & 5 & 5 & \mathrm{~V}\end{array}$

$\begin{array}{lllllllllll}0.2 & 0.5 & 5 & 2 & 3 & 0.2 & 0.7 & 1 & 5 & 5 & \mathrm{VI}\end{array}$

$\begin{array}{lllllllllll}0.2 & 0.5 & 5 & 2 & 1 & 1.0 & 0.7 & 1 & 5 & 5 & \mathrm{VII}\end{array}$

$\begin{array}{lllllllllll}0.2 & 0.5 & 5 & 2 & 1 & 0.2 & 7.0 & 1 & 5 & 5 & \text { VIII }\end{array}$

$\begin{array}{lllllllllll}0.2 & 0.5 & 5 & 2 & 1 & 0.2 & 0.7 & 5 & 5 & 5 & \text { IX }\end{array}$

$\begin{array}{lllllllllll}0.2 & 0.5 & 5 & 2 & 1 & 0.2 & 0.7 & 1 & 7 & 5 & X\end{array}$

$\begin{array}{lllllllllll}0.2 & 0.5 & 5 & 2 & 1 & 0.2 & 0.7 & 1 & 5 & 6 & \mathrm{XI}\end{array}$

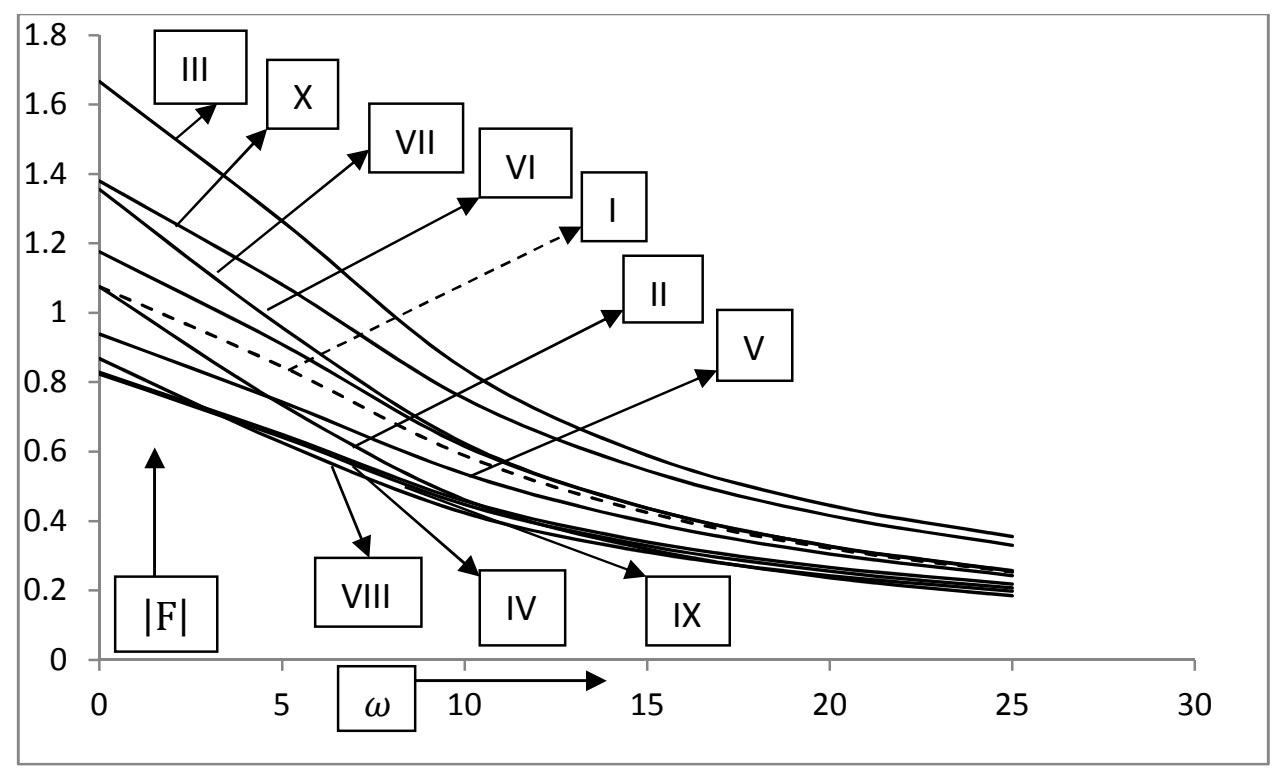

Fig. 3a. Amplitude of skin friction.

Table 2. Values of parameters plotted in Figs. $3 a \& 3 b$.

$\begin{array}{llllllll}Y & \lambda & \mathrm{Gr} & \mathrm{MH} & \mathrm{K} & \operatorname{Pr} & \mathrm{N} & \mathrm{A}\end{array}$

$\begin{array}{lllllllllll}0.2 & 0.5 & 5 & 2 & 1 & 0.2 & 0.7 & 1 & 5 & 1(---)\end{array}$

$\begin{array}{lllllllllll}0.3 & 0.5 & 5 & 2 & 1 & 0.2 & 0.7 & 1 & 5 & \text { II }\end{array}$

$\begin{array}{llllllllllll}0.2 & 1.0 & 5 & 2 & 1 & 0.2 & 0.7 & 1 & 5 & \text { III }\end{array}$

$\begin{array}{lllllllllll}0.2 & 0.5 & 1 & 2 & 1 & 0.2 & 0.7 & 1 & 5 & \text { IV }\end{array}$

$\begin{array}{llllllllll}0.2 & 0.5 & 5 & 3 & 1 & 0.2 & 0.7 & 1 & 5 & \mathrm{~V}\end{array}$

$\begin{array}{llllllllll}0.2 & 0.5 & 5 & 2 & 3 & 0.2 & 0.7 & 1 & 5 & \mathrm{VI}\end{array}$

$\begin{array}{llllllllll}0.2 & 0.5 & 5 & 2 & 1 & 1.0 & 0.7 & 1 & 5 & \mathrm{VII}\end{array}$

$\begin{array}{llllllllll}0.2 & 0.5 & 5 & 2 & 1 & 0.2 & 7.0 & 1 & 5 & \text { VIII }\end{array}$

$\begin{array}{llllllllll}0.2 & 0.5 & 5 & 2 & 1 & 0.2 & 0.7 & 5 & 5 & \text { IX }\end{array}$

$\begin{array}{llllllllll}0.2 & 0.5 & 5 & 2 & 1 & 0.2 & 0.7 & 1 & 7 & X\end{array}$ 


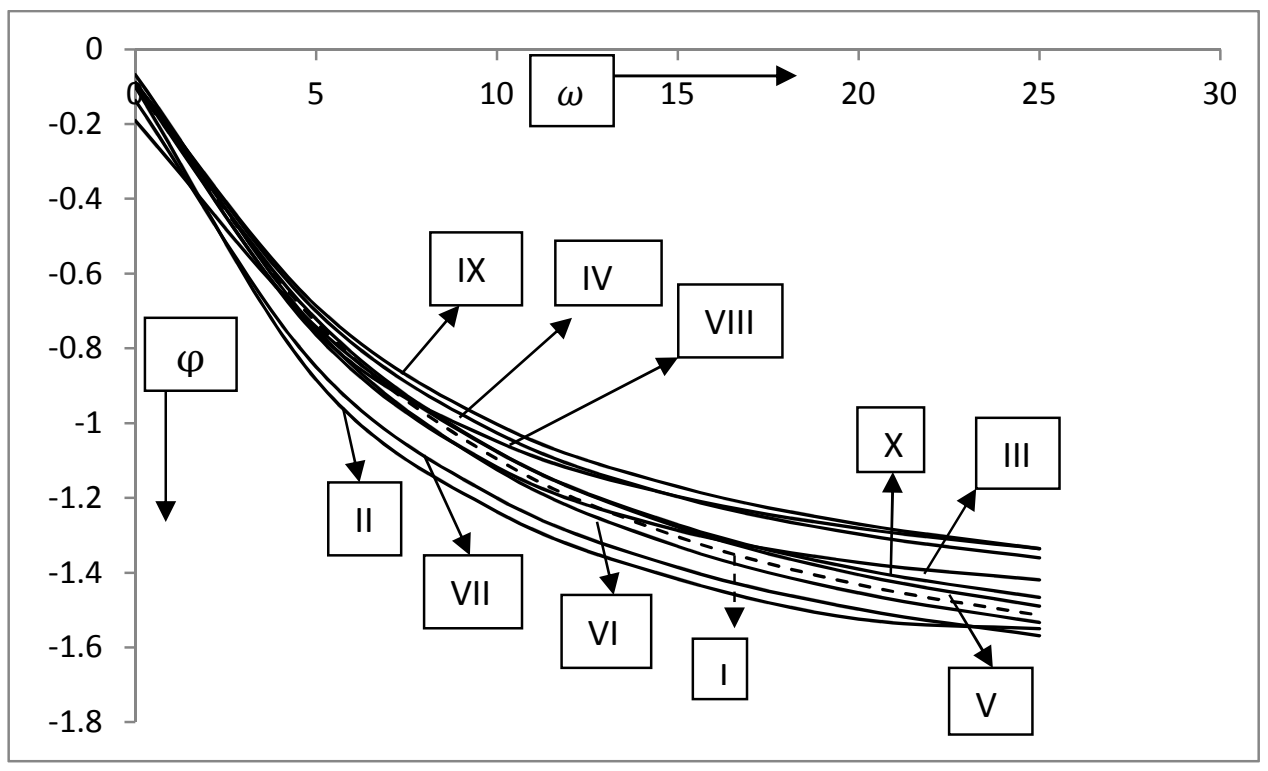

Fig. 3b. Phase of the skin friction.

\section{CONCLUSIONS}

An oscillatory hydromagnetic convective flow of viscous incompressible and electrically conducting fluid in a vertical porous channel is investigated. A closed form solution of the problem is obtained. It is found that the velocity remains parabolic over the width of the channel. The velocity increases with the increase of the injection/suction parameter, Grashof number, Hall Parameter and the pressure gradient. However, the velocity decreases with the increase of radiation parameter, Prandtl number and the frequency of oscillation. The amplitude increases with the increase of injection/suction parameter $\lambda$, the Grashof number Gr, and the pressure gradient parameter A. There is always a phase lag of the skin friction.

\section{NOMENCLATURE}

$\begin{array}{ll}\text { A } & \text { a constant } \\ \mathrm{B}_{0} & \text { magnetic field applied } \\ \mathrm{c}_{\mathrm{p}} & \text { specific heat at constant pressure } \\ |\mathrm{F}| & \text { amplitude of the skin-friction } \\ \mathrm{g} & \text { gravitational force } \\ \mathrm{Gr} & \text { Grashof number } \\ \mathrm{H} & \text { Hall parameter } \\ \mathrm{k} & \text { thermal conductivity } \\ \mathrm{K} & \text { permeability of porous medium } \\ \mathrm{M} & \text { Hartmann number } \\ \mathrm{N} & \text { heat radiation parameter } \\ \mathrm{p} & \text { pressure } \\ \mathrm{Pr} & \text { Prandtl number } \\ \mathrm{t} & \text { time variable } \\ \mathrm{T} & \text { fluid temperature } \\ \mathrm{T}_{1}, \mathrm{~T}_{2} & \text { plates temperatures }\end{array}$

$\mathrm{u}, \mathrm{v}, \mathrm{w}$ velocity components along $\mathrm{X}, \mathrm{Y}, \mathrm{Z}$ directions

$\mathrm{w}_{0} \quad$ injection/suction velocity

$\mathrm{x}, \mathrm{y}, \mathrm{z}$ variables along $\mathrm{X}, \mathrm{Y}, \mathrm{Z}$-directions

\section{Greek symbols}

$\alpha \quad$ mean radiation absorption coefficient

$\beta \quad$ coefficient of volume expansion

$\Gamma \quad$ viscoelastic parameter

$\lambda$ injection/suction parameter

$\omega$ frequency of oscillations

$\vartheta_{1} \quad$ kinematic viscosity

$\rho \quad$ fluid density

$\tau_{L} \quad$ skin-friction at the left wall

$\varphi \quad$ phase angle of the skin-friction

$\theta_{0} \quad$ mean non-dimensional temperature

* $\quad$ superscript representing dimensional quantities 


\section{References:}

[1] Alagoa, K.D., Tay, G., AbBey, T.M., Radiative and free convective effects of a MHD flow through a porous medium between infinite parallel plates with time-dependent suction. Astrophysics and Space Science 260 (1999) 455-468.

[2] Aldoss, T.K., Al-Nimr, M.A., Jarrah, M.A., Al-Shaer, B., Magnetohydrodynamic mixed convection from a vertical plate embedded in a porous medium. Numerical Heat Transfer A 28 (1995) 635.

[3] ARIEL, P.D., The flow of a viscoelastic fluid past a porous plate. Acta Mech. 107(1994) 199-204.

[4] AtTiA, H.A., Ewis, K.M., Unsteady MHD Couette flow with heat transfer of a viscoelastic fluid under exponential decaying pressure gradient. Tamkang J. Sci. And Engng. 13 (2010) 359-364.

[5] AtTia, H.A., Hall current effects on velocity and temperature fields of an unsteady Hartmann flow. Can. J. Phys. 76 (1998) 739.

[6] AtTia, H.A., Unsteady Hartmann flow of a viscoelastic fluid considering the Hall effect. Canadian J. Of Physics 82 (2004) 127.

[7] AtTIA, H.A., Unsteady MHD Couette flow of a viscoelastic fluid with heat transfer. Kragujevac J. Sci. 32 (2010) 5-15.

[8] Aтtia, H.A., Котв, N.A., MHD flow between two parallel plates with heat transfer. Acta Mechanica 117 (1996) 215-220.

[9] Chaudhary, R.C., Kumar Jha, A., Heat and mass transfer in elastico-viscous fluid past an impulsively started infinite vertical plate with Hall effect. Latin American Applied Research 38 (2008) 17-26.

[10] Choudhury, R., Jyoti Das, U., Heat transfer to MHD oscillatory viscoelastic flow in a channel filled with porous medium. Physics Research International 2012 (2012) 1-5. DOI: 101155/2012/879537.

[11] Cogley, A.C.L., Vinvent, W.G., GILES, E.S., Differential approximation for radiative heat transfer in Non-linear equations grey gas near equilibrium. American Institute of Aeronautics and Astronautics 6 (1968) 551-553.

[12] Coleman, B. D., Noll, W. An approximation theorem for functional, with applications in continuum mechanics. Archive for Rational Mechanics and Analysis, 6 (1960) 355-370.

[13] Cowling, T.G., Magnetohydrodynamics. Interscience Publications Inc., New York, USA, 1957.

[14] Hassanien, I.A., Mansour, M.A., Unsteady magnetic flow through a porous medium between two infinite parallel plates. Astrophysics and Space science, 163 (1990): 241-246.

[15] Hayat, T., Asghar, S., Siddiqui, A.M., Periodic unsteady flows of a non-Newtonian fluid. Acta Mech. 131 (1998) 169-173.

[16] Hossain, M.A., RASHID, R.I.M.I. Hall effect on hydromagnetic free convection flow along a porous flat plate with mass transfer. J. Phys. Soc. Japan 56 (1987) 97-104. 
[17] Kumar, R., CHAND, K., Effect of slip-conditions and Hall current on unsteady MHD flow of a viscoelastic fluid past an infinite porous plate through porous medium. Int. J. of Engineering Science and Technology (IJEST) 3(4) (2011) 3124-3133.

[18] Markovitz, H., Coleman, B.D., Incompressible second order fluids Advances in Applied Mechanics 8 (1964) 69-101.

[19] MeYER, R.C., On reducing aerodynamic heat transfer rates by MHD techniques. $J$. Aerospace Sci. 25 (1958) 561-563.

[20] Nigam, S.D., SingH, S.N., Heat transfer by laminar flow between parallel plates under the action of transverse magnetic field. Quarterly Journal of Mechanics and Applied Mathematics 13 (1960) 85-97.

[21] POP, I., GoRLa, R.S.R., Second order boundary layer solution for a continuous moving surface in a non-Newtonian fluid. Int. J. Engng. Sci. 28 (1990) 313-322.

[22] Rajgopal, K.R., A note on unsteady unidirectional flows of a non-Newtonian fluid. Int. J. Non-linear Mech. 17 (1982) 369-373.

[23] Rajgopal, K.R., Gupta, A.S., An exact solution for the flow of a non-Newtonian fluid past an infinite porous plate. Meccanica 19 (1984) 158-160.

[24] Rajgopal, K., Veena, P.H., Pravin, V.K., Oscillatory motion of an electrically conducting viscoelastic fluid over a stretching sheet in saturated porous medium with suction/blowing. Mathematical Problems in Engng. 1 (2006) 1-14.

[25] RAPTIS, A., PERDiKIS, C.P. (1985): Oscillatory flow through a porous medium by the presence of free convection flow. Int. J. Engng. Sci. 23 51-55.

[26] Raptis, A., Massalas, C., TzIVANidis, G. Hydromagnetic free convection flow through a porous medium between two parallel plates. Physics Letters A, 90 (1982) 288-289.

[27] SINGH, K.D., Effect of injection/suction on convective oscillatory flow through porous medium bounded by two vertical porous plates. Int. J. of Physical and Mathematical Sciences (IJPMS) 2 (2011) 140-147.

[28] Singh, K.D., Viscoelastic mixed convection MHD oscillatory flow through a porous medium filled in a vertical channel. Int. J. Physical and Mathematical Sciences 3 (2012) 194-205.

[29] SINGH, K.D., MHD mixed convection visco-elastic slip-flow through a porous medium in a vertical porous channel with thermal radiation. Kragujevac J. Sci., 35 (2013) 27-40.

[30] SINGH, K.D., GARG, B.P. Radiative heat transfer in MHD oscillatory flow through porous medium bounded by two vertical porous plates. Bull. Cal. Math. Soc. 102 (2010) 129-138.

[32] Singh, K. D., Chand, K., Sharma, S., Heat and Mass transfer in an unsteady MHD free convective flow through a porous medium bounded by vertical porous channel. Int. J. Math. Sci. \& Engng. Appl. 6 (2012) 317-336.

[33] SoundalgeKar, V.M., Hall and ion-slip effects in MHD Couette flow with heat transfer. IEEE Transactions on Plasma Science PS-7 (1979) 178.

[34] Soundalgekar, V.M., Bhat, J.P., Oscillatory channel flow and heat transfer. Int. J. Pure and Applied Mathematics 15 (1971) 819-828.

[35] SoundalgeKar, V.M., UPleKar, A.G., Hall effects in MHD Couette flow with heat transfer. IEEE Transactions on Plasma Science PS-7 (1986) 579. 
[36] VAJRAVElu, K., Exact periodic solution of a hydromagnetic flow in a horizontal channel. J. of Applied Mechanics 55 (1988) 981-983. 